\title{
Exploratory Visualization of Multivariate Data with Variable Quality *
}

\author{
Zaixian Xie \\ Shiping Huang \\ Matthew O. Ward \\ Computer Science Department \\ Worcester Polytechnic Institute \\ $\{$ xiezx,shiping,matt,rundenst $\} @$ cs.wpi.edu
}

Elke A. Rundensteiner

\begin{abstract}
Real-world data is known to be imperfect, suffering from various forms of defects such as sensor variability, estimation errors, uncertainty, human errors in data entry, and gaps in data gathering. Analysis conducted on variable quality data can lead to inaccurate or incorrect results. An effective visualization system must make users aware of the quality of their data by explicitly conveying not only the actual data content, but also its quality attributes. While some research has been conducted on visualizing uncertainty in spatiotemporal data and univariate data, little work has been reported on extending this capability into multivariate data visualization. In this paper we describe our approach to the problem of visually exploring multivariate data with variable quality. As a foundation, we propose a general approach to defining quality measures for tabular data, in which data may experience quality problems at three granularities: individual data values, complete records, and specific dimensions. We then present two approaches to visual mapping of quality information into display space. In particular, one solution embeds the quality measures as explicit values into the original dataset by regarding value quality and record quality as new data dimensions. The other solution is to superimpose the quality information within the data visualizations using additional visual variables. We also report on user studies conducted to assess alternate mappings of quality attributes to visual variables for the second method. In addition, we describe case studies that expose some of the advantages and disadvantages of these two approaches.
\end{abstract}

Keywords: Uncertainty visualization, multivariate visualization, data quality.

Index Terms: H.5.2 [Information Interfaces and Presentation]: User Interfaces-Graphical user interfaces

\section{INTRODUCTION}

The validity of decisions made and information extracted from exploratory visualization is, in a large part, dependent on the quality of the underlying data. The term data quality in this paper denotes the degree of uncertainty for the data. High quality indicates that the data is of high certainty and reliability. The variability of data quality has many causes and manifestations, including data accuracy, completeness, certainty, consistency, or any combination of these. It can include statistical variations or spread, errors and differences, minimum-maximum range values, noise, or missing data [15]. Visualization of data with variable quality and uncertainty has been identified as a critical research area in recent publications on future directions for visualization research $[20,10]$.

Information visualization is an increasingly important technique for the exploration and analysis of large datasets. Visualization takes advantage of the immense power, bandwidth, and pattern

\footnotetext{
*This work is supported under NSF grants IIS-0119276, IIS-00414380 and a grant from the NSA.
}

recognition capabilities of the human visual system. However, such power is limited by the visualization itself, that is, the conclusions drawn from the graphic representation are at best as accurate as the visualization. Therefore, to maintain the integrity of visual data exploration it is important to design a visualization so as to convey not only the actual data but also its quality [2].

In recent literature, we can find a number of research activities focused on visualizing quality attributes of data. They generally fall into two aspects, missing values and uncertainty visualization. XGOBI [18] and MANET [22, 7] are two visualization tools designed to deal with missing values. Uncertainty visualization is a very active research area that started in the GIS community $[11,24,16,9]$ and extended to other forms of data [5, 14]. However, prior work has primarily focused on spatio-temporal or univariate data. Little research has been reported on conveying data quality information within multivariate visualization techniques, which is surprising given how common multivariate data is in a wide range of applications.

Communication of potentially large and complex amounts of quality attributes for multivariate data presents a significant challenge. First, we need an appropriate model for quality measures, with the consideration that different data records, dimensions, or attribute values can have different levels or types of quality. Second, we need to find ways of incorporating quality information into the visual exploration process without overwhelming the analyst. As a motivating example of the need for conveying quality information, refer to Figures 1 and 2, which present an adaptation of the cars dataset [17]. We generated Figure 1 using traditional parallel coordinates without quality information. Figure 2 conveys the quality of records by means of the color of polylines. Red and orange polylines indicate that the corresponding records are of high or moderate confidence. In contrast, green lines correspond to records of low confidence. By ignoring green lines denoting records with low quality, Figure 2 reveals a relationship among the three dimensions: the more cylinders a car has, the lower its MPG and the higher its horsepower. Such a pattern is difficult to extract from Figure 1 , since the quality of the data is not shown.

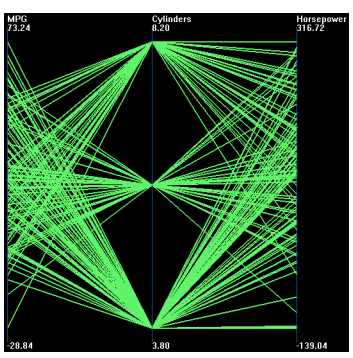

Figure 1: Parallel coordinates without quality information (the dataset is adapted from cars)

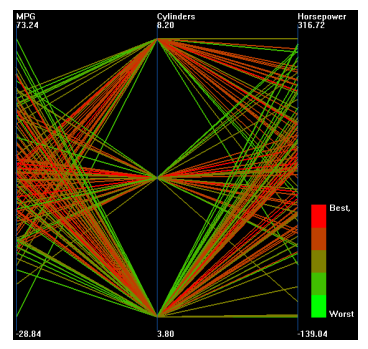

Figure 2: Parallel coordinates with quality information (record quality is mapped to the color of polylines)

The primary contributions of this paper include: 
- Developing a framework to define quality measures: Regarding the issue of data quality modeling, we define quality measures for multivariate data at three granularities, namely data value quality, record quality and dimension quality.

- Extending datasets with quality information as new dimensions: We construct new datasets that embody data value quality and record quality as new dimensions along with the original data records. In this way, we can use existing multivariate visualization techniques to convey quality information.

- Encoding quality information into the visualization of data: We incorporate data quality attributes into several multivariate data displays to convey this meta-information to users when they explore data.

- Evaluation of mappings from quality measures to visual variables: We present the results of a user study to compare the capabilities of different visual variables to convey quality attributes.

The remainder of this paper is organized as follows: In Section 2 existing techniques for data quality modeling, missing values visualization, and uncertainty visualization are reviewed. In Section 3 we propose a model of quality measures and two general approaches to visualizing quality measures. Section 4 describes the sample datasets used to demonstrate our techniques. Section 5 and 6 are dedicated to the discussion of two types of techniques to superimpose quality attributes on traditional multivariate data visualizations. Section 7 compares the two approaches and shows their advantages and disadvantages. Section 8 describes the user studies we performed. We conclude this paper in Section 9 with discussions and possible future research directions.

\section{RELATED WORK}

Data quality issues have been studied by many different research communities. Several important topics related to our work, including the definition and modeling of uncertainty, missing data visualization, and uncertainty visualization, are briefly discussed.

A report [19] by NIST divided uncertainty into two categories named Type A and Type B, which Olston and MacKinlay [14] called statistical uncertainty and bounded uncertainty. The former defines the uncertain value by a peak value and a potential distribution, while the latter gives a precise lower and upper bounds to convey the uncertainty. It is common to use a scalar value to present the certainty degree [24,5], although more complex representations are possible.

XGOBI [18] and MANET (Missing Are Now Equally Treated) $[22,7]$ are data visualization and analysis tools designed to handle missing data. Estimated values for the missing fields are generated by statistical inference algorithms. They then present graphic displays where the missing fields are replaced by the estimated values with indicators (e.g. different colors or positions) attached to show that values for those fields had been missing. The techniques, however, are generally limited to univariate or bivariate data.

Data uncertainty is a facet of data quality that has been studied in many fields, including the GIS community. The NCGIA initiative on "Visualizing the Quality of Spatial Information" [3] discussed the components of data quality, representational issues, the development and maintenance of data models and databases that support data quality information, and evaluation of visualization solutions in the context of user needs and perceptual and cognitive skills. After the NCGIA initiative, a flurry of activities have focused on uncertainty definition, modeling, computation and visualization [9, 11]. Within visualization, different practices in terms of graphical variable mappings have been tested. Use of color, hue, texture, fog and focus in static rendering of uncertainty and use of animation, flashing alternatively between data and its uncertainty, have been discussed in [3].

Wittenbrink, Pang, and Lodha [24] proposed a number of glyphs for visualizing uncertainty found in vector fields. Many mappings of uncertainty degree to glyph attributes were developed and evaluated. This work was greatly expanded in [16], where they describe techniques such as adding glyphs, adding geometry, modifying geometry, modifying attributes, animation, sonification, and psychovisual approaches.

Cedilnick and Rheingans [5] took a somewhat different approach, in that they embedded certainty information into annotations such as grid lines on displays. Distortions in the width and shape of these lines conveyed the level of certainty in the data in different regions of the data space while still allowing users to see the underlying data. Another unusual approach was reported by Brown [4], who used vibration of attributes such as hue, luminance, and vertex position to convey uncertainty.

These techniques are primarily directed toward spatio-temporal or univariate data and to date have not been applied to multivariate data. We will not only propose a general framework to fill this gap, but also present and evaluate two approaches in detail.

\section{Modeling of Data Quality and General Ap- PROACHES}

\subsection{Data Structure for Quality Measures}

As a reasonable starting point, we employed scalar values to measure uncertainty. While more complex representations can be imagined, we felt this basic assumption would be adequate in many, if not most, applications. We also noted that each data value, each record and each dimension might have an associated uncertainty measure. For example, occasional human error on data entry results in uncertainty of a single value; uncooperative participants in a survey may generate entire records of low confidence; a defective sensor might make a specified attribute highly unreliable in the whole dataset. Therefore, we assumed that quality measures consist of a vector of values for the record quality (one entry per record), a vector for the dimension quality (one entry per dimension), and a two dimensional table of values for the data value quality (one entry per value in the original dataset). All values for quality measures are normalized to the range of zero (lowest quality) to one (perfect quality). Figure 3 shows the configuration of these three types of data quality.

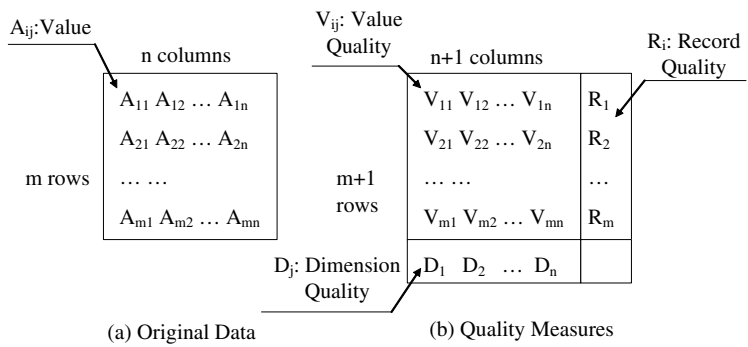

Figure 3: The structure of data quality defined in this paper

\subsection{Combining Quality Measures with the Original Dataset}

Obviously, quality measures can also be stored in tabular format, so we can visualize them using techniques applied to multivariate data. We could just use two separate multivariate visualizations, one for the data, and the other for the quality attributes. This, however, makes it difficult to see relationships between data and its quality. Instead, we can extend our dataset to include value and record quality as new dimensions. In other words, we combine the two tables 
shown in Figure 3 except the bottom line of table b. For example, if the original dataset has $n$ dimensions, the augmented dataset now has $2 n+1$ dimensions, with the additional $n+1$ dimensions used for $n$ columns of value quality and one column of record quality. Therefore, we can convey the quality information together with the original dataset if we apply existing multivariate visualization techniques to this quality-extented dataset.

\subsection{Encoding Quality Attributes in Data Visualization}

Another alternative approach is to convey quality measures using the graphical attributes of visual elements in existing multivariate visualizations. For example, the width of a polyline in parallel coordinates can represent the record quality of the corresponding data point. To conceptualize our approach, we will first define two functions and one operator, and then give an algorithm to describe the general process.

$\mathbf{G}(v, x, f): \quad v$ is a visual variable, $x$ is a numerical value representing a quality measure, and $f$ is a mapping function from a quality measure to the visual variable. This function returns a graphical attribute corresponding to $x$ in terms of the given visual variable and mapping function. For example, if $v$ is the line width, and $f$ maps one (perfect quality) to the thickest width and zero (lowest quality) to the thinnest width, the result will be a line width that is used to denote a quality measure.

$\operatorname{Draw}(\mathbf{o b}, G)$ : Draw the object $o b$ with $\mathrm{G}$ as its graphical attribute (set). For example, if $o b$ is a line, $G=\left\{G_{1}, G_{2}\right\}, G_{1}$ is a specific color, and $G_{2}$ is a specific line width, then this function will draw the line with the specific color and width.

$+_{g}$ : This operator returns the union of two graphical attributes.

Based on the above definition, the pseudo-code in Figure 4 shows a normal approach used by most multivariate visualization methods. Here $m$ is the number of records, $n$ is the number of dimensions and $v_{1}$ is the visual variable to denote attributes of records. For example, $v_{1}$ might be the length of ray axes in star glyphs. The last three lines (8-10) are only used for some of multivariate visualization techniques. For example, in parallel coordinates, we need them to draw the axes. Starting from this pseudo-code, we can derive our general approach, as shown in figure 5, to incorporate quality information into normal multivariate visualizations. Note that the three underlined steps, line 5, 7, and 11, are to combine graphical attributes from Figure 4 with new ones corresponding to the three types of quality measures, namely $V_{i j}, R_{i}$ and $D_{j}$, in the algorithm. $v_{2}, v_{3}$ and $v_{4}$ are visual variables to convey data value quality, record quality and dimension quality respectively. $f_{2}, f_{3}$ and $f_{4}$ are the corresponding mapping functions.

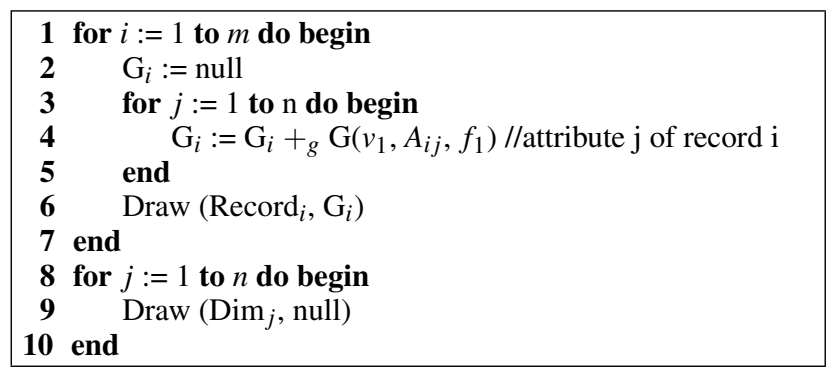

Figure 4: Normal approach used in multivariate visualization

To make the resulting visualization clear and help users interpret the data and its quality, two variations to the process described in Figure 5 are possible. First, in some situations, the user may only be

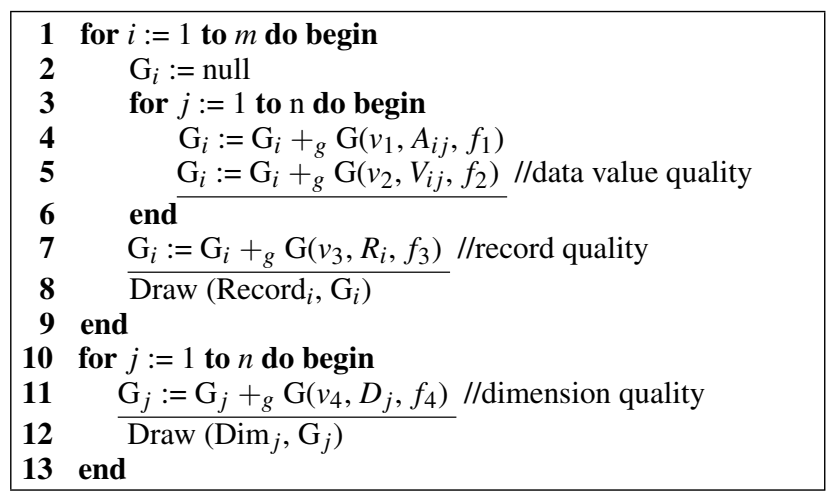

Figure 5: Our general approach to incorporating quality information

interested in a subset of the three quality types. The user may also at times feel that it is difficult to extract useful information when the visualization becomes overloaded with excessive information. We provide an interaction GUI to disable the visualization of any of the three quality types. Second, for situations where only a single type of quality information is to be conveyed, we investigate redundant mappings, where the quality information is mapped to more than one visual variable. This is important in some mappings where ambiguous interpretation is possible (e.g., depth in 3-D versus color).

\section{Sample Datasets}

It is difficult to find existing datasets augmented with a wide range of quality characteristics. However, we can easily collect some datasets with missing values from popular repositories. Starting from these datasets, the quality measures can be derived by imputation algorithms when the missing values are replaced with synthetic values. Below is the detailed description.

We employed a multiple imputation algorithm $[1,8]$ to generate estimated values for missing values. This algorithm repeats the imputation process more than once, producing multiple complete data sets until the estimates converge. Therefore, we can get $n$ values for each missing value, namely $y_{k}(1 \leq k \leq n)$, if we repeat the imputation process $n$ times. The formula to calculate data value quality for this missing value is given by

$$
V=1.0-\frac{\delta_{y}}{X_{\max }-X_{\min }}
$$

Here $X_{\max }$ and $X_{\min }$ are the maximum and minimum values of the dimension on which the missing value is. $\delta_{y}$ is the standard deviation of $y_{k}(1 \leq k \leq n)$. If the initial estimated value is close to the final imputed value, $\delta_{y}$ is small and $V$ is close to 1.0. It shows that the imputed value is reliable. Otherwise, the imputed value is not reliable. If $\delta_{y}>X_{\max }-X_{\min }$, we need to set the quality measure to 0.0 arbitrarily.

In this paper, we selected the datasets Echocardiogram and $\mathrm{Au}$ tomobile from [13]. Both have several missing values. The former describes patients that suffered heart attacks at some point in the past by some pathologic parameters. The latter presents the relationship between average loss payment per insured vehicle year and other characteristics of cars. We only show a subset of dimensions in Figure 6 and 9 to make it easy to find patterns related to quality.

To present the effectiveness of our approaches, we employed an alternative method, namely adding noise, to create datasets with quality measures starting from some real datasets without missing values. We add some random real numbers to a subset of data values in the dataset, and then compute the data value quality by inverse proportion to the absolute value of the noise. Note that the final value quality measure should be normalized to the range $[0,1]$. 
The datasets to which we applied adding noise include a time series dataset hipel-mcleod [17], a botanical dataset iris [6], and a product dataset cars [17], respectively. Hipel-mcleod presents the relationship among precipitation, temperature and daily flow of two rivers during a whole year. The attributes in iris are petal and sepal sizes for the iris flowers. The dimensions of cars describe the attributes of cars, including MPG, horsepower, the number of cylinders, and several others. Figures 1 and 2 in Section 1 only show the first three dimensions of this dataset to demonstrate our motivations more clearly.

Based on the value quality measures we obtained using the above two methods, we compute record quality and dimension quality measures by the following formulas:

$$
R_{i}=\frac{\sum_{j=1}^{m} V_{i j}}{m}, D_{j}=\frac{\sum_{i=1}^{n} V_{i j}}{n}
$$

Here $n$ is the number of records, $m$ is the number of dimensions, $R_{i}$ is the quality measure of the record $i$, and $D_{j}$ is the quality measure of the dimension $j$. Note that many ways exist to compute these quality attributes. Our goal was not to develop metrics, but to show how they could be incorporated into visualizations.

\section{Augmenting Datasets With Quality Information AS NeW Dimensions}

In this section, we show our approach to conveying quality information by embodying data value quality and record quality into the original dataset as new dimensions. We focus on three existing visualization techniques, namely parallel coordinates, scatterplot matrices and star glyphs. We identified several tasks that users might wish to perform when exploring datasets with variable quality. This list can help us design more effective visualizations:

- High quality data task: Focusing on the data that is of high quality (value or record) to draw conclusions with high confidence.

- Quality-data relationship task: Determining the relationships between quality measures and the original data.

- Quality information task: Obtaining information about the quality measures themselves, such as range and distribution.

We can visualize the quality-extended dataset directly using multivariate data visualizations. However, some of our prior research $[25,12]$ inspired us to do further processing on the final data mapping.

- Dimension masking : We can make some quality dimensions invisible to show only those in which users are interested. For example, we can show value quality only for dimensions in which it varies significantly.

- Dimension interleaving : Yang et al. [25] added two additional axes for each original dimension to present the degree of dissimilarity for a single data item in a dimension cluster. Two new axes were used to show the minimum and maximum of the corresponding dimension clusters for every data point. We borrowed this idea and regarded the value quality as an associated axis of the corresponding data dimension axis. We also put each value quality dimension close to its corresponding data axis. This variation can help analysts obtain the relationships between data values and their value quality.

- Interactive brushing : Martin and Ward [12] introduced and implemented N-dimensional brushing for XmdvTool. Based on this technique, we can easily implement a quality brush on the quality-extended dataset. The brush can extend across all of the value quality dimensions, or focus on record quality. Thus analysts can easily draw more reliable conclusions by, for example, selecting only records of high quality.

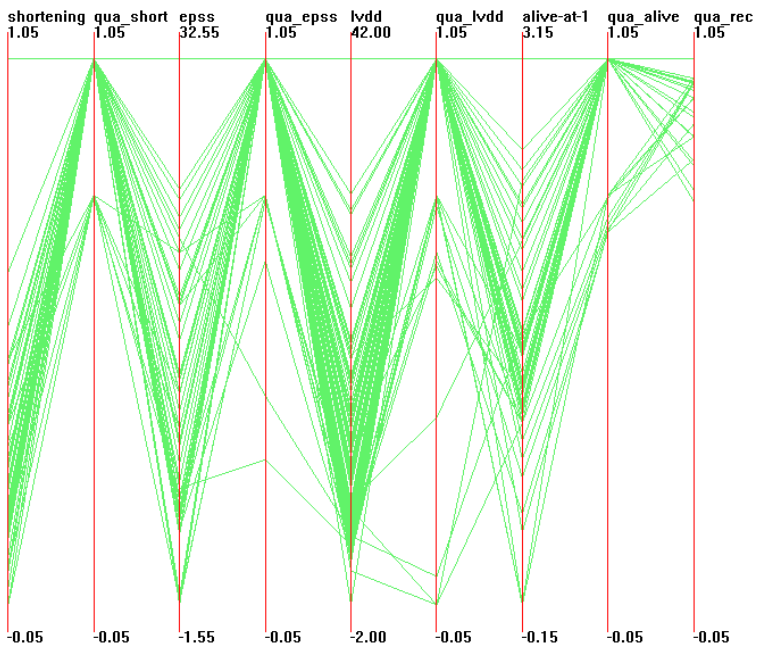

Figure 6: Enlarged dataset with quality measures visualized using parallel coordinates and dimension reordering(dataset:an adaptation of Echocardiogram)

Figure 6 shows the dimension interleaving technique on the quality-extended dataset. Each value quality axis is put beside the corresponding data dimension axis. The last axis is record quality. In this figure, we can find some patterns among the quality measures. Most of records have perfect value quality measures. Several lower value quality measures only exist on dimensions epss and $l v d d$. Moreover, the lower value quality measures normally correspond to lower value on the original dimensions. An evident limitation of this technique is that the figure cannot show any data correlation between different dimensions anymore.

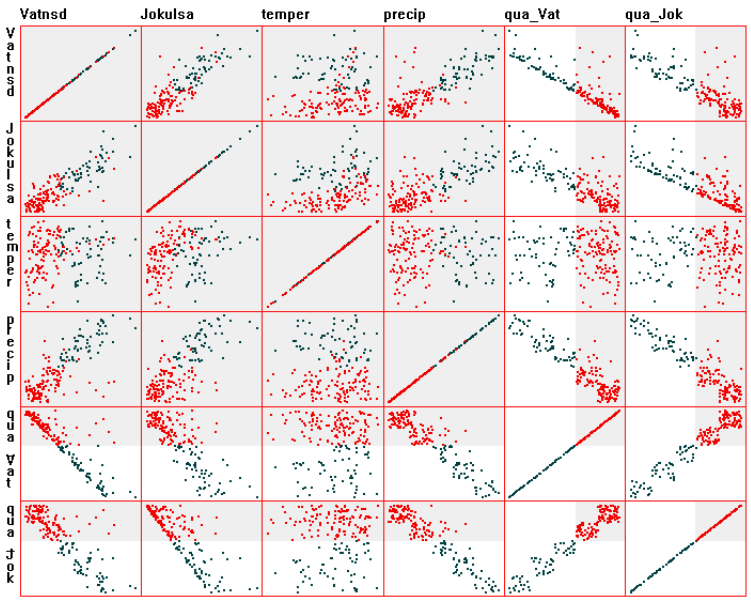

Figure 7: Interactive quality brushing (dataset:an adaptation of hipelmcleod, red point: selected data point)

In Figure 7, we constructed an N-dimensional brush across all of value quality axes and the record quality axis to select data points with high confidence. We masked two value quality axes and the record quality axis to save space for the data display. Since high confidence data points are marked in red, we can easily observe that 
high flow only occurs in times of high temperature, by focusing on red points. We can also note some patterns about the quality measures. For example, low flow data on dimensions Vatnsd and Jokulsa tends to be of high data value quality.

\section{InTEgrating Quality AtTRibutes in Data Visualiza- TIONS}

In this section, we discuss techniques to integrate quality attributes in three multivariate visualization methods. First, we show the underlying principles for selecting visual variables, and then present some effective configurations to map quality types to visual variables. We draw some conclusions about strengths, weaknesses, and limitations of the particular configurations or visual variables.

\subsection{Selection of Visual Variables}

Since we wish to embed the quality information in graphical attributes of existing visualizations, the selection of visual variables is one of the key factors in determining whether the visualization can enable users to interpret the quality information and draw reliable conclusions quickly. We first describe some strategies for selecting visual variables based on studies in perception, and then show our analysis.

Starting from perception theory [23] and the tasks identified in section 5, we consider the following criteria:

- Preattentive processing : Since we hope users will be able to easily identify data with high quality, we should use visual variables that are preattentively processed.

- Integral-separable dimension pairs : In visualization, a popular technique is to employ two or more graphical attributes of a visual object to represent different attributes of an actual object. The concept of integral-separable visual dimensions tells us whether one display attribute will be perceived independently from another. With integral graphical attributes, different attributes of a visual object are perceived holistically and not independently. On the contrary, with separable graphical attributes, people tend to make separate judgment about each graphical dimension [23]. In our general approach as shown in Figure 5, the visual variables $v_{1}, v_{2}$ and $v_{3}$ for quality attributes may each be attached to the same visual element. It is necessary to make any two of them as separable dimension pairs, so people are able to make separate judgments on data values, associated value quality and record quality.

- Monotonicity : Normally, monotonic display variables should be used to convey scalar values used to present quality measures. For example, most hue sequences (rainbow, red-green) are not monotonic, and thus we should not use hue for the quality-data relationship tasks or quality information tasks identified in Section 5. However, for the high quality data task, since users only focus on data with high quality, display variables without monotonicity also can work well.

A complete list of graphical attributes that are preattentively processed can be found in [23]. Some of them are not suitable for the multivariate visualization methods we used, such as line orientation and curvature. After considering the availability of each visual variable, we obtained a list we can use for conveying either data values or quality attributes : size (length, width), blur, color (hue, saturation, brightness), and position (2D, 3D). Since the degree of preattentive processing depends on the context [23], extensive evaluations need to be performed to identify the most effective visual variables.

Actually, integrality-separability is described as a continuum [23]. Regarding the list we derived above, we have some separable dimension pairs, including position (2D, 3D) and size, position and color (hue, saturation, brightness), 2D position and the third dimension, color and size. However, $\mathrm{x}$-size and $\mathrm{y}$-size, along with any two of the three parameters of color (hue, saturation, brightness) are most integral. We should avoid using the last two combinations. In addition, some of our experiments indicate that the third dimension can cause difficulties in observing data in parallel coordinates and scatterplot matrices because of serious occlusion.

To date, we have identified several available visual variables for each multivariate data visualization (Table 1). Note that the items marked with stars are not monotonic and thus are not generally suitable for the quality-data relationship or quality information tasks.

\begin{tabular}{|l|l|}
\hline Visualization & Visual Variables \\
\hline $\begin{array}{l}\text { Parallel Co- } \\
\text { ordinates }\end{array}$ & $\begin{array}{l}\text { Line width, Blur*, Hue*, Saturation, } \\
\text { Brightness }\end{array}$ \\
\hline $\begin{array}{l}\text { Scatterplot } \\
\text { Matrices }\end{array}$ & $\begin{array}{l}\text { Point size, Blur*, Hue*, Saturation, Bright- } \\
\text { ness }\end{array}$ \\
\hline Star Glyphs & $\begin{array}{l}\text { Line width, Blur*, Hue*, Saturation, } \\
\text { Brightness, 2D position, 3D position }\end{array}$ \\
\hline
\end{tabular}

Table 1: The available visual variables for each visualization technique

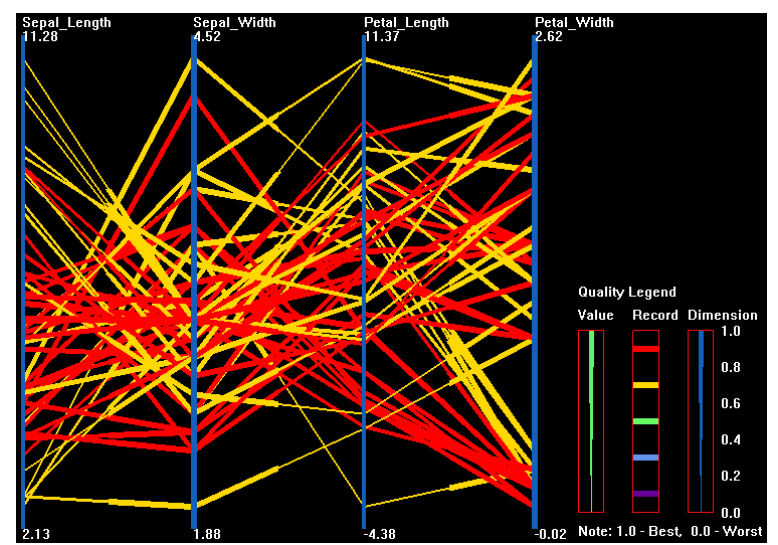

Figure 8: Sample of parallel coordinates (dataset:an adaptation of iris, value quality:line width, record quality:hue, dimension quality:line width)

\subsection{Visualizing Quality on Existing Multivariate Visual- ization Techniques}

In parallel coordinates, we map record quality and dimension quality to the graphical attributes of polylines and vertical axes respectively. To represent the value quality of one record for dimension $j$, we choose the section of the polyline near dimension $j$ corresponding to this record, and then use the graphical attributes of this section to convey the value quality. It is evident that we cannot map record quality and value quality to the same visual variable. Figure 8 shows an example. In this figure, the record quality is mapped to hue, while value quality and dimension quality are both mapped to line width. We can see the roughly positive correlation between dimension Petal_Length and Petal_Width if we focus on red lines or thick lines (records and values with high quality). It is difficult to draw such a conclusion without the data quality information.

For scatterplot matrices, we map dimension quality to graphical attributes of the diagonal plots. We map record quality and value quality to two graphical attributes of the points. One attribute represents record quality, the other represents the value quality of the dimension controlling the horizontal coordinate. In Figure 9, the hue of the points is used to convey value quality, while point size 
represents record quality and the saturation of diagonal plots denotes dimension quality. Here we used the adaptation of the dataset Automobile. We can clearly see that the dimension normalizedlosses has some imperfect values in yellow color that result from missing values. These values occurs when dimensions engine-size, horsepower and price have high values. We can draw an interesting conclusion that values of normalized-losses will often be unavailable when engine-size, horsepower or price has higher values.

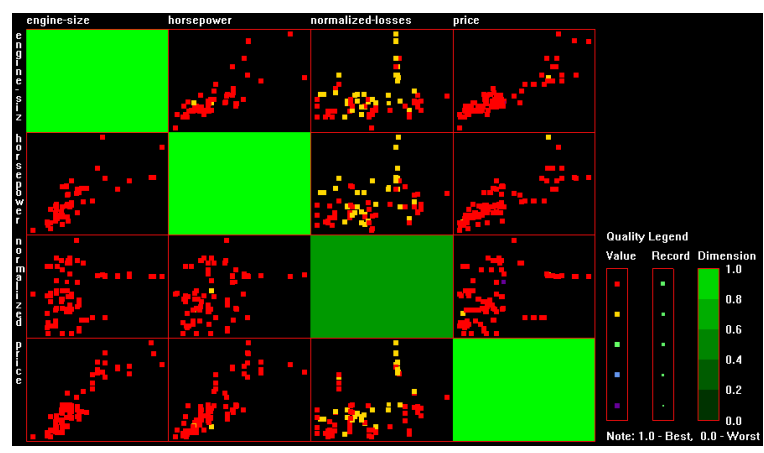

Figure 9: Sample of scatterplot matrices (dataset:an adaptation of Automobile, value quality:hue, record quality:point size, dimension quality:saturation)

On star glyphs, we mapped value quality to graphical attributes of the ray axes, and record quality to graphical attributes of the glyph perimeter or the whole glyph. We present two examples of mapping quality to star glyphs. In Figure 10, the line width and brightness convey the value quality and the record quality, respectively. From this figure, we can see that some dimensions almost always have low value quality(thin ray axes), but others do not. In Figure 11, we employ a redundant mapping, with the depth and hue both conveying the record quality. We can easily obtain the distribution of record quality measures from this figure.

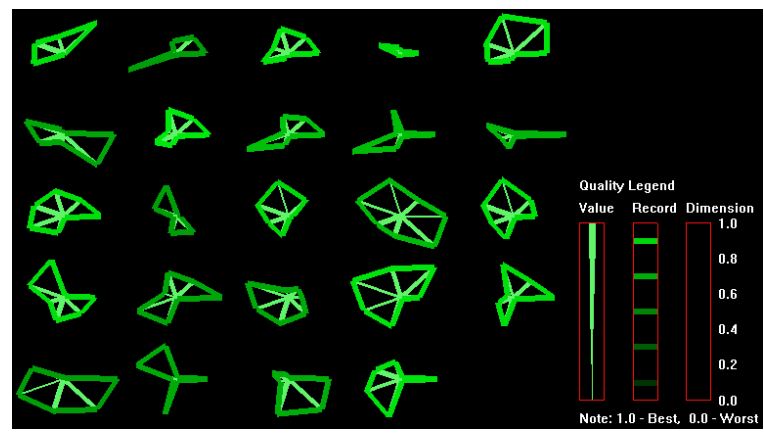

Figure 10: Sample of star glyphs (dataset:an adaptation of cars, value quality:line width, record quality:brightness )

\subsection{Analysis}

Using several datasets, we studied the strengths and weaknesses of each several mappings based on combinations of the selected visual variables. We concluded that:

- Line width, hue, saturation, and brightness can be used for conveying dimension quality in either parallel coordinates or scatterplot matrices.

- We can easily integrate data value and record quality into normal visualizations when we map quality measures to hue, saturation and brightness, even though hue is not monotonic.

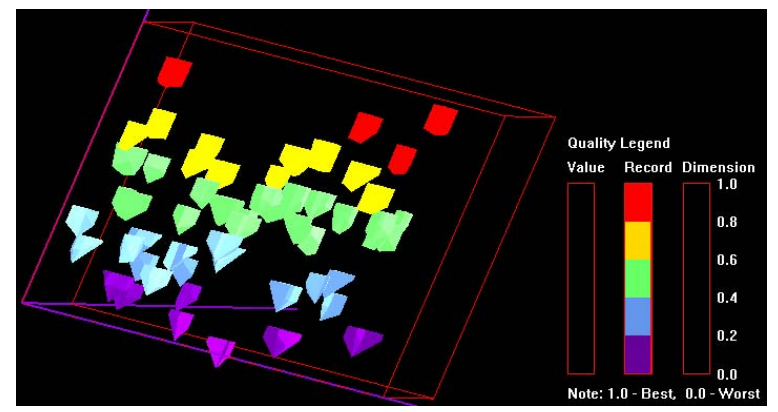

Figure 11: Sample of star glyphs (dataset:an adaptation of cars, the record quality is mapped to the third dimension and hue )

The generated figures in this case can convey the quality information in an easily interpreted manner. The advantage is that they do not require extra space. In contrast, line width and point size need additional space. Therefore, we found both of them are only suitable for relatively small datasets. Significant occlusion will occur if we visualize large datasets.

- In parallel coordinates and scatterplots, It is easier to interpret quality information when value quality and record quality are visualized in separate visualizations. Otherwise when the graphical attributes of value quality and record quality are shown together, polylines and points are overloaded with too much quality information.

- Because the techniques we were testing are inherently 2-D, the visualizations can include more information by mapping the quality to the third spatial dimension. In some camera positions, we can discover interesting patterns. For instance, we can clearly see the clustering and distribution of record quality from Figure 11. Since ambiguity is often inevitable in 3-D viewing, redundant mapping can help us to obtain a consistent interpretation. However, when the record quality values mostly fall in a narrow range, such as $(0.8,1.0)$, large datasets will cause significant occlusion in the 3-D mapping.

\section{Comparison between the Two Approaches}

In this section, we will compare the two approaches to incorporating quality information into multivariate data visualizations, namely the $\mathrm{ND}$ (new dimensions) method in Section 5 and the VE(visual encoding) method in Section 6. Regarding the tasks identified in Section 5, the advantages and disadvantages of the two methods are evident. First, for the high quality data task, the ND method employs interactive brushing and the VE method makes use of visual variables to highlight the data points with high quality to enable analysts to draw conclusions of high confidence. Since brushing techniques normally use color to highlight data in which users are interested, it can be regarded as a special visual variable that conveys only values one (selected records having high confidence) or zero (unselected records having low confidence). Imagine that one user used the quality brush to select data points with record quality falling into the range $[0.8,1]$, but then felt that the quality range was too narrow and wanted to extend it to $[0.6,1]$. Now he must redefine the brush. It is not a flexible solution compared with the VE method, since the VE method marks different levels of quality measures with different levels of a graphical attribute (e.g. color), and reconfiguration is not necessary in the above situation. However, the user must face the challenge of distinguishing different levels of graphical attributes corresponding to different quality measures within the VE method. In contrast, the advantage of the ND method is that it is easy to distinguish the records of high reliability and low reliability since they are just different colors in our 
implementation of interactive brushing. Therefore, we must consider the trade-off between ease of observation and flexibility of the two approaches to performing the high quality data task. Second, the ND method is a better solution than the VE method regarding the quality-data relationship task and quality information task. Since quality measures and the original data are both regarded as dimensions in the quality-extended dataset, the existing multivariate visualizations obviously are powerful tools for determining the relationships between quality measures and the original data or obtaining information about the quality measures themselves.

Another important topic is the trade-off between information overload and data-ink ratio [21]. Using the VE method, the data-ink ratio is more than two times the original visualizations. However, it is more difficult for users to retrieve this information, especially for parallel coordinates and scatterplot matrices, because both value quality and record quality are mapped to the same line or point. For the ND method, the data-ink ratio is unchanged, but users do not need face the challenge to distinguish too much information in a single visualization, as in the VE method.

The number of data dimensions is also an important factor in determining which method we should use. As we know, most multivariate data visualizations do not scale well to datasets with a large number of dimensions. For a fixed configuration (display device and users), there is always an upper limit for the number of dimensions. With the ND method, this limit must be divided by two. If dimension masking is employed, the limit is less severe.

\section{Evaluation}

We described how to integrate quality attributes into three types of visualizations and examined some samples in Section 6. Some mappings enable users to easily solve quality-related tasks; however it is harder in other mappings. Here we present a user study we carried out to attempt to determine the visual variables on parallel coordinates and scatterplot matrices that can convey quality information most effectively.

\subsection{Experiment Design}

The effectiveness of visualizations with quality information depends on the mapping and properties of datasets. Therefore, we designed questions for our user studies based on the combination of visual variables and the number of records in datasets. To avoid testing too many mappings, we experimented informally with a larger number of mappings and then selected a subset of these for formal evaluation. We designed six groups of questions. Each group corresponded to one mapping. The six mappings we tested were line width, brightness, and hue for parallel coordinates, and dot size, brightness, and hue for scatterplot matrices. Each group had two subsets of questions. One used small datasets having 50 records, which we labeled small. The other employed bigger datasets having 200 records, which we labeled moderate. In each subset, we designed two classes of questions. One required users to estimate the percentage of records with a specific record quality or value quality range. This belongs to the quality information task identified in Section 5. The other required users to classify the relationship between two dimensions by ignoring those records with low quality. This belongs to the high quality data task. The relationships were limited to positive and negative correlations for simplicity.

We used a similar method as the one we introduced in Section 4 to construct datasets for this experiment. Since the relationships between dimensions of actual datasets are normally complex, instead of positive and negative correlations, we used artificial datasets here. In artificial datasets, we constructed positive or negative relationships between adjacent dimensions.

Sixteen graduate students from the Computer Science Department at WPI participated in this experiment. Twelve of them were not familiar with parallel coordinates or scatterplot matrices. All participants were required to read the instructions before answering questions.

\subsection{Experiment Result and Analysis}

Figure 12 shows the response accuracy (RA) on the small dataset and moderate datasets for each mapping. Furthermore we calculated response times (RT) of participants. However, we noticed that RT is often affected by the experiment environment. For instance, we observed that some participants spent much more time reading instructions than others. Moreover, we did not find significant difference of RT for different mappings. Therefore, we focused on the analysis of RA. Theoretically, a high RA indicates that a mapping method enables easy retrieval of data quality information.

First, we compared the RA of different visual variables in the same mapping configuration (visualization methods and sizes of datasets) using a paired samples t-test. Statistical results revealed the difference among capabilities of visual variables as we expected. On parallel coordinates, when datasets are small, hue had a significantly higher RA than line width $(p<0.05)$. The mean RA of hue is bigger than brightness, although we cannot find significant difference between hue and brightness $(p=0.17)$. The reason is possibly due to a higher degree of preattentive processing for hue than line width and brightness under this configuration. When datasets become larger, more significant differences existed between hue and line width $(\mathrm{p}<0.001)$. On scatterplot matrices, we find that point size had a significantly higher RA than brightness when datasets are small $(\mathrm{p}<0.01)$. Although no significant difference exists between hue and brightness $(\mathrm{p}=0.13)$, the mean RA of hue is bigger than brightness. The possible reason is that the brightness of points is difficult to distinguish. Therefore, we concluded that brightness is not a good option for scatterplot matrices.

Second, we compared RA on datasets with different sizes. When we used line width on parallel coordinates, moderately sized datasets have significantly lower RA $(\mathrm{p}<0.01)$. Other visual variables do not result in significant difference between small sizes and moderate sizes. However, we note that mean RA of point size is significantly higher than brightness for scatterplot matrices $(\mathrm{p}<0.01)$ when datasets are small, and when datasets are moderate, the mean RA of point size former is slightly lower than brightness. Therefore, we suspect that the effectiveness of point size is also sensitive to the size of the dataset, since it also requires additional space, as in line width. The sizes of datasets that we used are not large enough to demonstrate this weak point of point size. In addition, we found a significant difference between line width and point size on moderate datasets $(\mathrm{p}<0.01)$, so we can conclude the point size can handle larger datasets than line width.

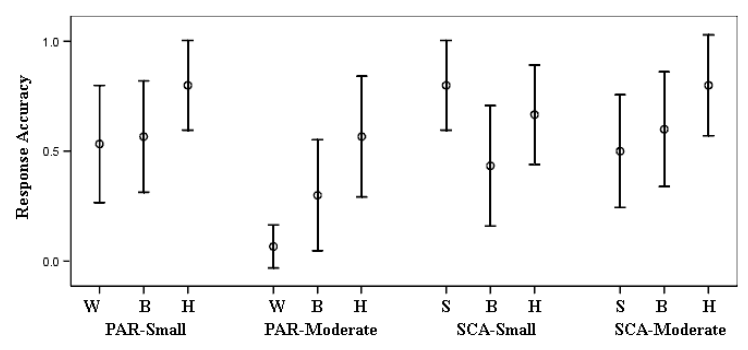

Figure 12: Response accuracy with 95\% confidence interval for different mappings (W: Line Width, B: Brightness, H: Hue, S: Point Size, PAR: Parallel Coordinates, SCA: Scatterplot Matrices, Small: The dataset having 50 records, Moderate: The dataset having 200 records)

From the above observations, we can derive some characteristics 
of the tested visual variables.

- Hue has a stronger capacity to convey quality information under parallel coordinates, not only for small datasets, but also for larger ones. The reason is probably that it has a highr degree of preattentive processing and does not need extra space.

- Point size has a better performance in conveying quality information than brightness under scatterplot matrices when datasets are small. But its capability becomes weak when datasets become larger. Brightness under scatterplot matrices is a bad option since it is difficult to distinguish. Hue is a fine option for scatterplot matrices when the dataset is larger, although it is not monotonic.

- The size of the dataset affects the capacity of line width significantly, but affects that of hue much less. We also suspect the effectiveness of point size under large datasets since it needs extra space like line width. But we can conclude that the sizes of datasets have a less serious influence on point size than line width.

Our conclusions are limited. More configurations should be employed in our experiments. For example, we can test larger datasets than the moderate datasets we used to test how the size of datasets affects the performance of different visual variables.

\section{Conclusions ANd Future Work}

In this paper, we have described the growing need to integrate data quality information into the visual process. We identified three important data quality types (record, dimension, data value) and presented two approaches to integrating quality information into data visualizations. One is an enlarged dataset containing quality measures as new dimensions. The other is to map quality information to visual variables not currently in use in existing multivariate visualization methods. We also analyzed the advantages and disadvantages of these two approaches. In addition, we performed an evaluation and showed the effectiveness of visual variables under different configurations.

There are many potential future directions for this work. Some that we are currently pursuing include:

- Continued experimentation and more strict user studies with different mappings of quality variables to visual variables using our current set of multivariate data visualizations.

- Expansion of the set of data visualizations to include other methods for visualizing multivariate data, such a pixeloriented methods.

- Investigation of other types of quality information encountered in exploratory visualization, such as structure quality (e.g., the certainty with which records are grouped within a hierarchical clustering).

This work can be applied to other attributes of data besides quality. For example, some data may have a security or risk attribute, and visually exploring data in this space might reveal some holes in a data security policy or highlight threats in risk analysis. Our belief is that information about the data can often be as important as the data itself.

\section{REFERENCES}

[1] P. D. Allison. Missing data. SAGE Publications, Thousand Oaks CA, 2002.

[2] R. Amar and J. Stasko. A knowledge task-based framework for design and evaluation of information visualizations. Proc. IEEE Symposium on Information Visualization, pages 143-150, 2004.
[3] M. K. Beard, B. P. Buttenfield, and S. B. Clapham. NCGIA research initiative 7: Visualization of spatial data quality. Technical report, National Center for Geographic Information and Analysis, 1991.

[4] R. Brown. Animated visual vibrations as an uncertainty visualisation technique. Proc. 2nd international conference on Computer graphics and interactive techniques in Australasia and Southe East Asia, pages 84-89, 2004.

[5] A. Cedilnik and P. Rheingans. Procedural annotation of uncertain information. In Proc. IEEE Symposium on Information Visualization, pages 77-84, 2000.

[6] DASL. The data and story library [http://lib.stat.cmu.edu/dasl]. Cornell University, 1996.

[7] H. Hofmann and M. Theus. Selection sequences in manet. Computational Statistics, 13(1):77-88, 1998.

[8] S. Huang. Exploratory visualization of data with variable quality. Master's thesis, Worcester Polytechnic Institute, 2004.

[9] G. J. Hunter. New tools for handling spatial data quality: Moving from academic concepts to practical reality. URISA Journal, 11(2):25-34, 1999.

[10] C. Johnson, R. Moorhead, T. Munzner, H. Pfister, P. Rheingans, and T. S. Yoo. NIH-NSF Visualization Research Challenges Report. IEEE Computer Society, Los Alamitos CA, 2006.

[11] A. M. MacEachren. Visualizing uncertain information. Cartographic Perspective, 13:10-19, 1992.

[12] A. Martin and M. Ward. High dimensional brushing for interactive exploration of multivariate data. Proc. of Visualization, pages 271$278,1995$.

[13] D. Newman, S. Hettich, C. Blake, and C. Merz. UCI repository of machine learning databases [http://www.ics.uci.edu/ mlearn/ mlrepository.html]. University of California, Irvine, Dept. of Information and Computer Sciences, 1998.

[14] C. Olston and J. D. Mackinlay. Visualizing data with bounded uncertainty. In Proc. IEEE Symposium on Information Visualization, pages 37-40, 2002.

[15] A. Pang. Visualizing uncertainty in geo-spatial data. report for a committee of the computer science and telecommunications board. Technical report, University of California, Santa Cruz, 2001.

[16] A. Pang, C. Wittenbrink, and S. Lodha. Approaches to uncertainty visualization. The Visual Computer, 13(8):370-390, 1997.

[17] StatLib. Statlib-datasets archive [http://lib.stat.cmu.edu/datasets]. Carnegie Mellon University, Dept. of Statistics, 1999.

[18] D. Swayne and A. Buja. Missing data in interactive high-dimensional data visualization. Computational Statistics, 13(1):15-26, 1998.

[19] B. N. Taylor and C. E. Kuyatt. Guidelines for evaluating and expressing the uncertainty of nist measurement results. Technical report, National Institute of Standards and Technology Technical Note 1297, 1994.

[20] J. J. Thomas and K. A. Cook. Illuminating the Path: The Research and Development Agenda for Visual Analytics. IEEE Computer Society, Los Alamitos CA, 2005.

[21] E. Tufte. The Visual Display of Quantitative Information. Graphics Press, Cheshire CT, 1982.

[22] A. Unwin, G. Hawkins, H. Hofmann, and B. Siegl. Interactive graphics for data sets with missing values - manet. Journal of Computaional and Graphical Statistics, 4(6):113-122, 1996.

[23] C. Ware. Information Visualization: Perception for Design. Morgan Kaufmann Publishers, San Francisco CA, second edition, 2004.

[24] C. Wittenbrink, A. Pang, and S. Lodha. Glyphs for visualizing uncertainty in vector fields. IEEE Transactions on Visualization and Computer Graphics, 2(3):266-279, 1996.

[25] J. Yang, M. O. Ward, E. A. Rundensteiner, and S. Huang. Visual hierarchical dimension reduction for exploration of high dimensional datasets. Joint Eurographics - IEEE TCVG Symposium on Visualization, pages 19-28, 2003. 